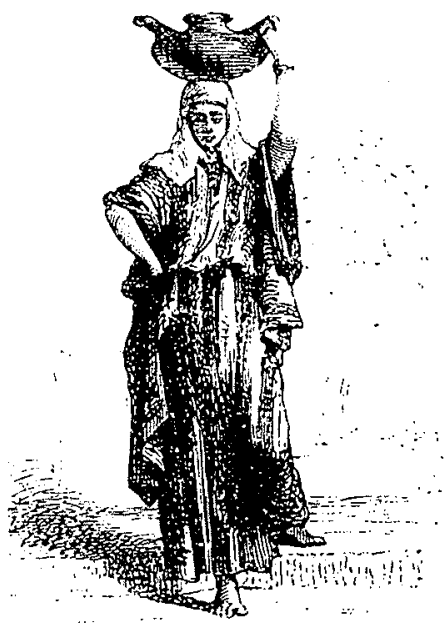

\title{
APPLICATION OF MOMENTUM EQUATION IN THE HYDRAULIC JUMP
}

\author{
BY N. S. GOVINDA RAO * AND RAMAPRASAD * *
}

This paper concerns the use of a well known method in solving the hydraulic jump. This method can be recognized as a particular case of the solution of the momentum integral equation used extensively in boundary layer problems. In solving the momentum integral equation for any boundary layer, a velocity distribution in the boundary layer satisfying all or some boundary conditions is assumed and is substituted in the equation. If the boundary layer is turbulent an additional assumption has to be made about the shear stress at the wall.

To apply the method to the problem of the hydraulic jump, we consider for simplicity a broad channel of rectangular section with a horizontal bed (Fig. 1). The momentum integral equation for this case can be written as :

$\int_{0}^{y_{2}} u_{2}^{2} d y-\int_{0}^{y_{1}} u_{1}^{2} d y=\frac{1}{2} g\left(y_{1}^{2}-y_{2}^{2}\right)$

While deriving this equation, the shear stresses at the bottom and sides of the channel have been neglected because their contribution to the resulting force on the water mass between sections I and II

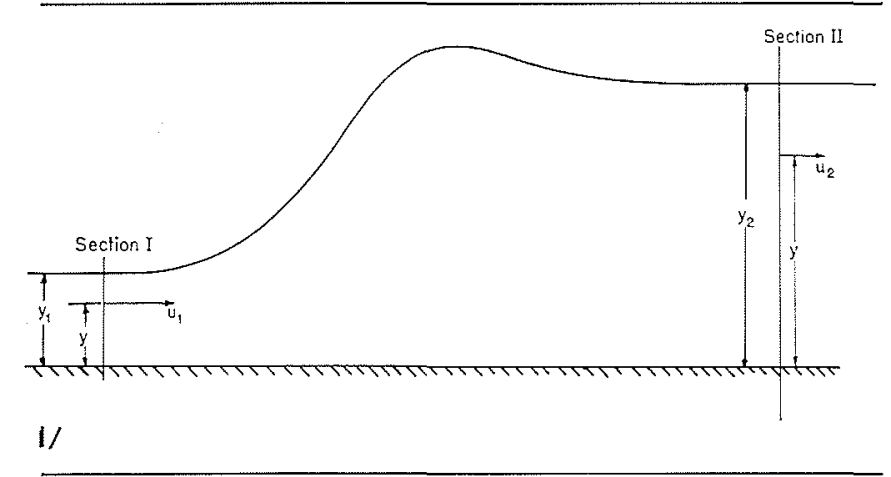

* Professor of Civil and Hydraulic Eng., Indian Institute of Science, Bangalore-12.

* Lecturer, Department of Civil and Hydraulic Eng, Indian Institute of Science, Bangalore-12. is very small compared to the rate of change of momentum of the water mass and so has little effect on it. In eq. (1), $u_{1}$ and $u_{2}$ denote the velocities of water at sections $I$ and II respectively at a height $y$ from the bottom of the channel.

The most usual procedure is to assume $u_{1}$ and $u_{2}$ have constant values. If the discharge per unit width of the channel is $q$, then :

$$
u_{1}=q / y_{1} \quad \text { and } \quad u_{2}=q / y_{2}
$$

Substituting these values in eq. (1) we get:

$$
\therefore q^{2}\left(\frac{1}{y_{2}}-\frac{1}{y_{1}}\right)=\frac{1}{2} g\left(y_{1}^{2}-y_{2}^{2}\right)
$$

which simplifies to :

$$
\left(\frac{y_{2}}{y_{1}}\right)^{2}+\left(\frac{y_{2}}{y_{1}}\right)-\frac{2 q^{2}}{g y_{1}^{3}}=0
$$

We shall denote the quantity $q^{2} / g y_{1}{ }^{3}$ henceforth in this paper by $F_{1}{ }^{2}$. Solving (3), we get the well known equation :

$$
\frac{y_{2}}{y_{1}}=\frac{1}{2}\left[\sqrt{1+8 \mathrm{~F}_{1}^{2}}-1\right]
$$

The assumption represented by eq. (2) is only one of many which can be made regarding the velocity distribution. The basis for the assumption of any distribution is commonly taken in boundary layer problems to be the boundary conditions which have to be satisfied by the distribution. The distribution (2), however, does not satisfy what is considered to be the most important condition of zero slip at the boundary (in this case at the channel bed).

We shall now examine the consequences of assuming velocity distributions satisfying various conceivable boundary conditions. We may, for example, seek to satisfy several or all of the following conditions : 


\begin{tabular}{|c|c|c|}
\hline \multicolumn{3}{|c|}{ Table } \\
\hline $\begin{array}{c}\text { VELOGITY } \\
\text { DISTRIBUTION } \\
u_{1}\end{array}$ & $\begin{array}{c}\text { BOUNDARY } \\
\text { COND- } \\
\text { ITIONS } \\
\text { SATIS- } \\
\text { FIED }\end{array}$ & $y_{2} / y_{1}$ \\
\hline A. $\quad \frac{q}{y_{1}}$ & $\begin{array}{l}(b),(c) \\
\text { and }(d)\end{array}$ & $\frac{1}{2}\left[\sqrt{1+8 F_{1}^{2}}-1\right]$ \\
\hline B. $\quad \frac{2 q}{y_{1}^{2}} \cdot y$ & $\begin{array}{l}(a),(c) \\
\text { and }(d)\end{array}$ & $\frac{1}{2}\left[\sqrt{1+10.6 \mathrm{~F}_{1}{ }^{2}}-1\right]$ \\
\hline C. $\frac{8}{7} \frac{q}{y_{1}}\left(\begin{array}{l}y \\
y_{1}\end{array}\right)^{1 / \pi}$ & $(a)$ & $\frac{1}{2}\left[\sqrt{1+8.13 \mathrm{~F}_{1}^{2}-1}\right]$ \\
\hline D. $\frac{3 q}{y_{1}^{2}} \cdot y-\frac{3}{2} \frac{q}{y_{1}{ }^{3}} \cdot y^{2}$ & $\begin{array}{l}(a) \\
\text { and }(b)\end{array}$ & $\frac{1}{2}\left[\sqrt{ } 1+9.6 \mathrm{~F}_{1}^{2}-1\right]$ \\
\hline E. $\frac{\pi}{2} \frac{q}{y_{1}} \sin \left(\frac{\pi}{2} \frac{y}{y_{1}}\right)$ & $\begin{array}{l}(a),(b) \\
\text { and }(c)\end{array}$ & $\frac{1}{2}\left[\sqrt{\left.1+9.9 \mathrm{~F}_{1}^{2}-1\right]}\right.$ \\
\hline F. $\frac{2.59 q}{y_{1}} \log _{e}\left(\frac{y}{y_{1}}\right)$ & $(a)$ & $\frac{1}{2}\left[\sqrt{ } 1+10.1 \mathrm{~F}_{1}^{2}-1\right]$ \\
\hline G. $\frac{-6 q}{y_{1}^{3}} y^{2}+\frac{4 q}{y_{1}^{4}} y^{3}$ & $\begin{array}{l}(a),(b) \\
\text { and }(e)\end{array}$ & $\frac{1}{2}\left[\sqrt{1+11.9} \mathrm{~F}_{1}^{2}-1\right]$ \\
\hline $\begin{array}{l}\text { H. } \frac{4 q}{y_{1}^{2}} y \\
\frac{4 q}{y_{1}^{3}} y^{2}+\frac{4 q}{3 y_{1}^{4}} y^{3}\end{array}$ & $\begin{array}{l}(a),(b) \\
\text { and }(d)\end{array}$ & $\frac{1}{2}\left[\sqrt{1+9.14 \mathrm{~F}_{1}{ }^{2}}-1\right]$ \\
\hline $\begin{array}{l}\text { J. } \frac{20 q}{y_{1}{ }^{4}} y^{3}- \\
\frac{30 q}{y_{1}^{5}} y^{1}-\frac{12 q}{y_{1}^{(3}} y^{5}\end{array}$ & $\begin{array}{l}(a),(b) \\
(c),(d) \\
\text { and }(e)\end{array}$ & $\frac{1}{2}\left[\sqrt{1+12.54 \mathrm{~F}_{1}-1}\right]$ \\
\hline
\end{tabular}

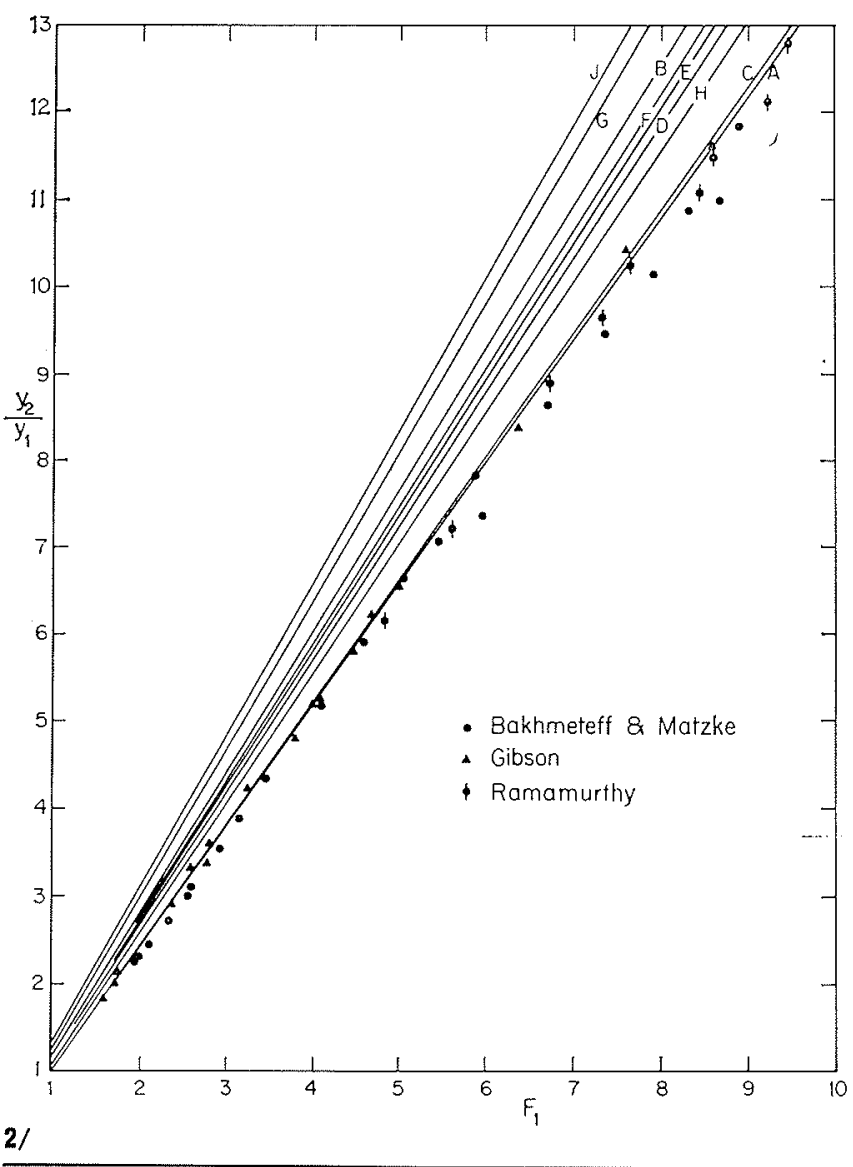

(a) $u_{1}=0$ and $u_{2}=0$ at $y=0$

(b) $\frac{d u_{1}}{d y}=0$ at $y=y_{1} ; \frac{d u_{2}}{d y}=0$ at $y=y_{2}$

(c) $\frac{d^{2} u u_{1}}{d y^{2}}=0$ at $y=0 ; \frac{d^{2} u_{2}}{d y^{2}}=0$ at $y=0$

(d) $\frac{d^{2} u_{1}}{d y^{2}}=0$ at $y=y_{1} ; \frac{d^{2} u_{2}}{d y^{2}}=0$ at $y=y_{2}$

(e) $\frac{d u_{1}}{d y}=0$ at $y=0 ; \frac{d u_{2}}{d y}=0$ at $y=0$

It is to be remarked here that no boundary condition written above, except for condition $(a)$, is assumed to be a priori true in the hydraulic jump. The object is only to investigate the result of satisfying some or all of the conditions set forth above.

Condition ( $a$ ) ensures that there is no slip at the channel bed. Conditions $(b),(c)$ and $(d)$ are analogous to those satisfied by the velocity distribution in a laminar boundary layer on a flat plate in the absence of pressure gradient. The boundary condition (e) should be satisfied if we consider that the boundary layer on the channel bed separates at the beginning of the jump due to the adverse pressure gradient (induced by the sudden rise in the water level) and re-attaches at its end.

The table above gives the different velocity distributions assumed, the boundary conditions which they satisfy and the resulting formula for the ratio of the water depths of the jump. The distribution of $u_{1}$ only is given, that for $u_{2}$ being obtained by substituting $y_{2}$ for $y_{1}$ in it.

The numerical coefficients of the distribution have been so adjusted that all the distributions satisfy in addition to the respective boundary conditions the equation :

$$
\int_{0}^{y_{1}} u_{1} d y=\int_{0}^{y_{2}} u_{2} d y=q,
$$

the discharge per unit width of the channel.

It will be noted that all distributions give rise to formulae of the type :

$$
\frac{y_{2}}{y_{1}}=\frac{1}{2}\left[\sqrt{1+a \mathrm{~F}_{1}^{2}-1}\right]
$$

The formulae (A) to (J) from the table are shown plotted in Figure 2. Experimental data from different sources are also shown in the figure. The figure shows that the formula (A), derived from a distribution which does not satisfy the most important zero slip boundary condition, gives the most satisfactory result whereas distributions satisfying the zero slip condition give formulae which are in varying degrees of error. Next to distribution (A), the most satisfactory result is given by distribution (C), which is the well known one-seventh power law extensively used in turbulent boundary layer problems. But even this distribution which satisfies the zero slip condition leads to a formula more in error than (A). Thus, although for practical purposes eq. (4) is admirably suited, its success, coupled with the failure of other formulae derived from apparently more suitable velocity distributions, calls for explanation. The answer to 
his question may have a bearing on the utility and reliability of the momentum integral equation method in solving many problems in hydraulics.

The momentum integral equation is not the only one by which a solution is likely to be obtained. The energy equation is difficult to derive because of the importance of turbulent friction as a contributor to the loss of energy. However, we can start with the moment of momentum equation, which expresses the fact that the rate of change of moment of momentum of the water mass between sections I and II (Fig. 3) about a traverse axis lying in the bed of the channel is equal to the moment of the external forces acting on this water mass. The rate of change of moment of momentum of the water mass considered about the point $\mathrm{P}$ can be easily proved to be :

$$
\int_{0}^{y_{2}} u_{2}^{2} y d y-\int_{0}^{y_{1}} u_{1}^{2} y d y
$$

The external forces acting on the water mass are : 1. the force $F_{1}$ due to water pressure on section I;

2. the force $F_{2}$ due to water pressure on section II;

3 . the weight $W$ of the water mass acting at its centre of gravity;

4. the reaction $\mathrm{R}$ from the bed acting at the centre of pressure at the base of the water mass.

For each of the distributions given in the previous table the expression (6) is identically zero. This is true for any distribution of the type given in the table. For, in all distributions of $u_{1}$ of that type, the coefficients of the powers of $y$ depend only on $q$ and $y_{1}$, due to the conditions to be satisfied. Thus $q, y$ and $y_{1}$ are the only variables affecting $u_{1}$, and it follows from dimensional analysis that a relation of the type :

$$
u_{1}=\frac{q}{y_{1}} f\left(\frac{y}{y_{1}}\right)
$$

should hold, $f$ being a function depending to some extent on the boundary conditions.

Hence,

$$
\begin{aligned}
\int_{0}^{y_{1}} u_{1}^{2} y d y & =\int_{0}^{y_{1}} \frac{q^{2}}{y_{1}^{2}} y\left[f\left(\frac{y}{y_{1}}\right)\right]^{2} d y \\
& =q^{2} \int_{0}^{1} \frac{y_{-}}{y_{1}}\left[f\left(\frac{y}{y_{1}}\right)\right]^{2} d\left(\frac{y}{y_{1}}\right) \\
& =c q^{2}
\end{aligned}
$$

where $c$ is a constant.

Similarly :

$$
\int_{0}^{y_{2}} u_{2}^{2} y d y=c q^{2}
$$

where $c$ has the same value as for

$$
\int_{0}^{y_{1}} u_{1}^{2} y d y
$$

Thus the expression (6) vanishes identically.

This is still the case if one supposes that $u_{1}$ is affected not only by $q, y_{1}$ and $y$, but by the kinematic viscosity and the bed shear stress as well. For, in that case, there will be a relation of the form :

$$
u_{1}=\frac{q}{y_{1}} \mathrm{~F}\left(\frac{y^{u} \tau}{\nu}, \frac{q}{\nu}, \frac{y}{y_{1}}\right)
$$

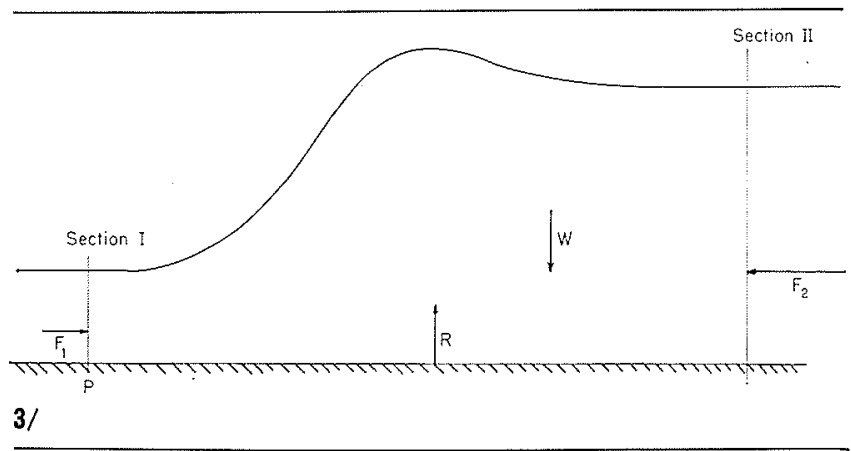

where $u_{\tau}$ is the friction velocity and $F$ is an arbitrary function, $u_{\tau}$ depends, again, on $q, y_{1}$ and $v$, so that $u_{1}$ ultimately depends on $q, y_{1}$ and $v$. Hence, the integral

$$
\int_{0}^{y_{1}} u_{1}^{2} y d y
$$

also depends on $q, y_{1}$ and $\nu$. So, by dimensional analysis, the integral is readily seen to be the form :

$$
q^{2} \varphi\left(\frac{q}{\nu}\right)
$$

In the same way,

$$
\int_{0}^{y_{2}} u_{2}^{2} y d y
$$

also reduces to (7) and hence expression (6) again vanishes identically.

This result means that the algebraic sum of the moments of the external forces on the water mass about $\mathrm{P}$ must be zero. On assuming hydrostatic pressure distribution on sections I and II, it can be easily shown that $F_{1}$ and $F_{2}$ give rise to an anticlockwise moment equal to :

$$
\frac{\rho}{6} g\left(y_{1}^{3}-y_{2}{ }^{3}\right)
$$

where $\rho$ is the density of water. Therefore the weight $W$ and the resultant bed reaction $R$ must together give rise to a clockwise moment equal to this magnitude. Hence $\mathrm{R}$ must be situated some distance to the left of $W$. This means that the pressure on the bed of the channel must be more than that due to hydrostatic pressure in the beginning of the jump and less towards the end. When this is the case, the centre of gravity of any element contained between two vertical sections separated by an infinitesimal distance must be accelerated upwards in the beginning of the jump and downwards towards the end. This means that the mean profile of the water surface in the jump must be as shown in Figures 2 and 3 and must not have a continuously rising form as is usually assumed.

\section{References}

The experimental data plotted in Figure 2 have been taken from the following references:

[1] BaknmetefF and Matzks. - Trans, ASCE, 101 (1936), p. 630 .

[2] Gubson (A.H.). - Proc. Instn. C.E., 197 (1913-14) pt. III, p. 233 .

[3] Ramanurthy (A.S.). - Thesis M. Sc., (II Sc.) (1960), p. 155. 